\title{
Euripide et l'imagination aérienne, textes réunis par Jacqueline Assaël
}

Lucie Thévenet

\section{(2) OpenEdition}

1 Journals

Édition électronique

URL : http://journals.openedition.org/kentron/923

DOI : $10.4000 /$ kentron.923

ISSN : 2264-1459

Éditeur

Presses universitaires de Caen

\section{Édition imprimée}

Date de publication : 31 décembre 2016

Pagination : 215-219

ISBN : 978-2-84133-840-5

ISSN : 0765-0590

\section{Référence électronique}

Lucie Thévenet, «Euripide et l'imagination aérienne, textes réunis par Jacqueline Assaël », Kentron [En ligne], 32 | 2016, mis en ligne le 10 mai 2017, consulté le 17 novembre 2020. URL : http:// journals.openedition.org/kentron/923; DOI : https://doi.org/10.4000/kentron.923

\section{(c) (i) (9)}

Kentron is licensed under a Creative Commons Attribution-NonCommercial-NoDerivatives 3.0 International License. 


\section{Euripide et l'imagination aérienne, textes réunis par Jacqueline Assaël, Paris, L'Harmattan (Thyrse; 6, Collection du CTEL), Université Nice Sophia Antipolis, 2015, 202 pages.}

Jacqueline Assaël, auteur d'ouvrages sur l'œuvre d'Euripide dans sa facette intellectuelle et philosophique ${ }^{1}$, s'est aussi intéressée, sans doute guidée par sa pratique poétique personnelle, à la question de l'inspiration poétique dans son ouvrage Pour une poétique de l'inspiration, d'Homère à Euripide (Louvain - Namur - Paris Dudley (MA), Peeters [Collection d'études classiques; 21], 2006), qui trouve dans le présent recueil une sorte de prolongement centré de nouveau sur Euripide, avec une exploration des images poétiques de ses pièces liées au ciel. Il est composé de sept articles, groupés en trois parties, dont la progression mène le lecteur de l'«esthétique de l'imagination aérienne d'Euripide» (trois articles), à sa «dramatique» (trois articles), et vient conclure sur le rapport au «tragique de la vision du monde d'Euripide» (deux articles). Le corpus des pièces est varié, incluant une pièce perdue, Andromède, et l'on notera que la majeure partie des analyses porte sur des passages lyriques, ce qui conforte l'association prônée entre imaginaire aérien et poésie.

Un avant-propos de Joël Thomas, spécialiste de l'imaginaire, met l'ouvrage sous l'égide de Gaston Bachelard et de Gilbert Durand, pour replacer l'imaginaire aérien au sein d'un réseau plus vaste: il propose, pour en tracer les grandes lignes, un tableau de synthèse associant éléments, figures parentales, figures archétypales, genres littéraires et symboles, et le place dans une chronologie de cycles temporels successifs : l'air est ainsi relié au monde diurne, au père, à l'épopée et à la tragédie, à la figure du héros, avec pour symbole le binôme ascension / chute. Le lien entre les images aériennes et le genre tragique, celui d'Euripide en particulier, se trouve donc expliqué par le « désir rêvé de l'élévation et la hantise fantasmée de la chute» (p. 11), ce qui rejoint la problématique aristotélicienne du héros tragique oscillant entre ces deux pôles. La notion de ciel ne peut se penser sans celle, complémentaire, de terre, ni celle du haut sans le bas. Or, l'intérêt du traitement du thème chez Euripide vient, semble-t-il, du mélange entre l'imaginaire «héroïque» propre à la tragédie et un imaginaire plus « romanesque», qui serait la marque d'une sorte d'étape, du passage d'un monde à un autre, quand «les forces de l'anankè et du fatum s'éloignent au profit de la petite musique de la psychè individuelle» (p. 15). Euripide est donc bien un "poète entre deux mondes», et le traitement de l'imaginaire ascensionnel qui le caractérise en est la marque.

1. Cf. Intellectualité et théâtralité dans l'œuvre d'Euripide, Nice, Association des publications de la Faculté des lettres de Nice (Publications de la Faculté des lettres, arts et sciences humaines de Nice), 1993; Euripide, philosophe et poète tragique, Louvain - Namur - Paris - Dudley (MA), Peeters (Collection d'études classiques; 16), 2001. 
La première partie, comportant trois articles, est intitulée «Pour une esthétique de l'imagination aérienne d'Euripide». Dans le premier article, Juan Tobias Napoli travaille sur «Ekphrasis et persuasion: une vision aérienne dans la parodos d'Iphigénie à Aulis d'Euripide». Après quelques pages visant à définir les différents types d'ekphrasis, l'auteur s'attache, de façon peut-être un peu périphérique, à la description des troupes achéennes observées depuis une position en surplomb par les jeunes filles du chœur, une vision d'en haut sur le mode de la teichoscopie, qui confère, selon lui, un caractère aérien à la description. Dans le deuxième article, Christine Amiech explore «Deux chants aériens chez Euripide: l'un plus nostalgique (Iphigénie en Tauride, v. 1089-1151), l'autre plus allègre (Hélène, v. 1451-1511)». L'auteur se focalise sur Iphigénie en Tauride et Hélène, pièces jumelles où il n'est guère étonnant qu'avec deux héroïnes transportées par les dieux dans l'éther jusqu'en Égypte et en Tauride, Euripide ait eu recours aux images aériennes. C. Amiech replace ces deux exemples dans l'économie de chaque pièce, puis évoque de façon synthétique «de brèves allusions mythiques qui favorisent l'envol vers un ailleurs» (titre de partie), avec une étude signifiante de l'usage des sonorités liquides. Elle aborde ensuite «la poésie maritime qui élargit l'horizon et préfigure l'heureux dénouement», avec le motif de l'évasion pour un retour en Grèce par la voie maritime, poésie dans laquelle apparaît la métaphore de l'aile renvoyant aux voiles des navires. Elle termine sur «le point culminant de la poésie de ces deux chants: la poésie aérienne des oiseaux ", avec un judicieux rappel de la définition du poète dans le Ion platonicien, présenté comme "chose légère, ailée et sacrée». Le ballet des oiseaux est également à rapprocher de la danse du chœur, dans une lecture métathéâtrale des passages. L'image commune des ailes de l'imagination trouve ainsi son expression parfaite dans ces morceaux poétiques, subtilement reliés à l'intrigue par le motif de l'exil forcé des deux jeunes femmes qui aspirent à un retour dans leur pays. Nul doute que ces passages ont contribué au grand succès des chants choraux d'Euripide dans l'Antiquité, comme l'auteur le rappelle à dessein. Enfin, Ghislaine Jay-Robert se propose, dans un article intitulé «L'imagination aérienne d'Euripide vue par Aristophane», d'étudier la manière dont Aristophane présente Euripide à travers le prisme des images aériennes, ce qui permet en retour de s'interroger sur ce que ces choix révèlent d'Aristophane lui-même. Partant de la distinction entre l'air et l'éther, espace au-dessus de la terre et espace encore supérieur, l'auteur dégage un point crucial: les occurrences du terme éther n'apparaissent que dans certains types de passages, les passages de parodies de poésie tragique, dithyrambique, morceaux lyriques, etc. (p. 54), soit un style plus élevé, pourrait-on dire. L'éther est présenté comme représentatif d'Euripide - même s'il est également associé à Socrate ou à Agathon comme intellectuels adeptes des idées nouvelles. L'analyse des passages concernés montre qu'il s'agit là d'une manière de stigmatiser la poésie d'Euripide, dont la qualité légère (comme dans la pesée des vers des Grenouilles) et éthérée est 
vite, de positive, considérée comme négative, voire sert à montrer son inconsistance. Aristophane vise également l'amalgame entre le sublime et le trivial, donné comme caractéristique de la poésie d'Euripide, un mélange des registres que l'on retrouve par ailleurs dans le théâtre d'Aristophane lui-même, et qui lui permet de mettre en question le propre fonctionnement de son écriture.

La deuxième partie, «Pour une dramatique de l'imagination aérienne d'Euripide», porte sur l'intégration des thèmes aériens aux problématiques théâtrales, en particulier à l'opposition entre haut et bas envisagée dans l'espace scénique. Comme le rappelle Joël Thomas dans l'avant-propos, il existe un «clivage entre le terrestre et le céleste, l'humain et le divin, le pur et l'impur», qui se traduit dans le théâtre d'Euripide par la difficulté de l'accession au domaine supérieur aérien (p. 13-14).

Pierre Voelke, dans son article "Médée dans l'éther athénien », interroge le dénouement de la Médée, pièce dans laquelle l'héroïne apparaît en hauteur, sur un char tiré par des dragons: reprise ou non du code du deus ex machina, position exacte de Médée... Cette intervention se distingue du discours des divinités (ordre, dimension étiologique, annonce prophétique) tout en en reprenant certains codes. La question principale liée à la thématique aérienne est, selon l'auteur, celle de la destination: Athènes. Pour l'expliquer, il fait appel au troisième stasimon de la pièce, qui présente le peuple athénien comme relevant à la fois de la terre et de l'éther, appartenance terrestre par son origine autochtone et céleste par sa qualité d'enfant des dieux; s'y trouve associée l'image d'Aphrodite, grâce à son pouvoir de symbiose, par son rôle d'union entre la terre et le ciel. Cette vision des Athéniens est ensuite reliée à la description par Platon de l'ancienne Athènes dans le Timée et dans le Critias: même origine autochtone des Athéniens, et image de l'homme comme plante céleste décrite dans le Timée, avec une tête qui s'élève vers le ciel, auquel l'être humain est apparenté. Dans le cas du stasimon euripidéen, comme dans celui des discours platoniciens, la figure des Athéniens est aussi à lire comme double poétique et fictionnel du public. L'auteur revient ensuite à Médée: son départ n'est pas uniquement une fuite par l'éther vers les dieux, mais vers cette Athènes entre terre et ciel censée, d'après la promesse faite par Égée, pouvoir l'accueillir. Autre question de fond: cette mère infanticide est-elle un hôte envisageable pour Athènes? dans et hors de la fiction? Il apparaît que, si Médée la magicienne utilise

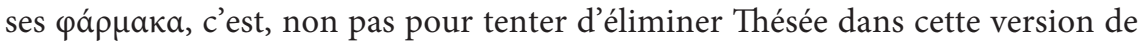
l'histoire, mais pour aider Égée à avoir une descendance, ce qui l'érige au rang de double de l'Aphrodite ouranienne, dans la figure dessinée par le deuxième stasimon, avec sa dimension terrible; l'auteur conclut sur le fait qu'elle devient ellemême une image poétique dématérialisée par le voyage vers l'éther. Ensuite, Maria Silvina Delbueno, dans son article intitulé «Images d'une antinomie: la dimension aérienne de l'impureté de Médée, d'Euripide, dans la résignification de Laurent Gaudé», conduit une étude comparée de la dimension de l'imagination aérienne 
du mythe de Médée au théâtre, dans la pièce d'Euripide et dans celle de Laurent Gaudé, Médée Kali. Médée y devient l'avatar de la déesse indienne Kali, comme elle une magicienne séductrice, mais aussi une pestiférée contagieuse, porteuse de souillure. La pièce commence à la fin de la pièce d'Euripide, après les meurtres, et le propos est de transférer les corps des enfants de Médée hors du sol grec, pour les ramener dans sa patrie. L'article passe en revue la question de la pureté de l'espace aérien d'Athènes et de l'impureté de Médée: on retrouve le troisième stasimon évoqué par l'article précédent, lié à l'air pur que viendrait souiller Médée. Sont par ailleurs étudiés le motif de la peste dans les deux pièces, la dialectique des espaces intérieurs et extérieurs, pour arriver à la dimension transgressive des deux figures féminines, marginales et liées à la souillure, et à l'examen de la pestilence de l'espace aérien à partir de l'infanticide, puis à partir du bûcher. La dimension aérienne y semble quelque peu périphérique, mais elle reste fondamentale pour la question de la transmission de la souillure. Pour clore cette partie, Silvia S. Reyes et Marcela A. Ristorto se proposent dans «Images aériennes dans les Bacchantes d'Euripide" d'étudier les images liées au registre aérien comme l'expression de la double nature de Dionysos, pour dégager la correspondance entre la nature ambivalente du dieu et celle des images aériennes. On retrouve l'opposition aérien/terrestre, humain / divin, et sa projection dans l'espace scénique du théologéion et du proskènion. Les auteurs englobent dans l'analyse l'imagination dynamique de l'air, avec trajets horizontaux et verticaux, et donc la gestuelle dionysiaque faite de mouvements du corps verticaux et horizontaux, ou, à l'inverse, de l'immobilité du sommeil. Ils y ajoutent les sons, véhiculés par l'air, et l'on connaît l'importance de la musique et des cris dans les rituels bacchiques. Ces images sont révélatrices de la double nature de Dionysos et de celle de ses fidèles (dualité des groupes de bacchantes, lydiennes et thébaines). L'article se poursuit avec l'analyse des aspects positifs, puis négatifs de Dionysos, pour se clore sur la réaffirmation de l'ambiguïté du dieu, lui-même terrible et doux.

La troisième partie est centrée sur "L'imagination aérienne et le tragique de la vision du monde d'Euripide». Tout d'abord, Jocelyne Peigney, avec «Mouvements du ciel et tournoiement des sorts chez Euripide: le tourbillon d'Hélène, les emboîtements des Phéniciennes", montre comment le topos de la variabilité du sort des hommes se trouve renouvelé chez Euripide grâce aux images de l'éther, des airs et des vents. À partir d'un passage de l'Hélène, v. 711-715, et en particulier

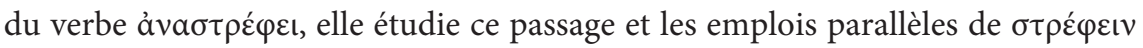
dans le corpus. L'idée de tourner, de tourbillon, est ensuite reliée à la description du typhon dans les Météorologiques d'Aristote (III, 1; 371a 9-15). L'idée de tourbillon des sorts vient renouveler le topos de la variabilité des sorts en y ajoutant l'image des tournoiements du monde, superposition du neuf et de l'ancien assez typique de la manière d'Euripide, non seulement dans son écriture littéraire, mais aussi dans son écriture musicale, que les chœurs de l'Hélène viennent explicitement 
commenter. La même figure de l'enroulement se trouve ensuite mise au jour dans les Phéniciennes: le passage où Jocaste, s'adressant à Étéocle, prend pour exemple la règle des échanges célestes (v. 541-548), la description des guerriers lors de la teichoscopie et celle des éléments figurant sur les boucliers au quatrième épisode. L'auteur conclut sur l'intertextualité avec Eschyle et la réécriture des Sept contre Thèbes, en étudiant le réinvestissement développé des éléments liés au ciel. Enfin, le dernier article est de la main de Jacqueline Assaël elle-même, qui s'intéresse à «L'Éther initiatique dans l'Andromède d'Euripide», en travaillant sur les fragments de cette pièce perdue. La dimension initiatique de la pièce se trouve confortée par l'étude des références aériennes. Le ciel est un élément important dans la pièce: Andromède enchaînée, immobilisée, l'évoque et l'invoque; il s'agit de l'espace lié à Persée, son sauveur, qui viendra par les airs, chaussé des sandales ailées d'Hermès; et, à terme, c'est le lieu qui les accueillera tous deux, lors de leur transformation en constellations (le katastérisme). Ce trajet faisant passer les personnages par des épreuves, dont ils sont délivrés pour parvenir à un destin immortel, calque celui de l'itinéraire initiatique. D'autres éléments viennent conforter la lecture: la présence de l'Écho comme forme dégradée de l'être, l'étude du vocabulaire "mystérique" lié au ciel, le parallèle avec le mythe platonicien de la caverne, en particulier la progression spatiale et symbolique vers la dissipation de l'illusion, vers le réel. L'auteur souligne également l'importance du rôle de l'Amour libérateur, lié à la pensée orphique, et la symbolique de la jeune fille assimilée au rocher, relevant donc du domaine terrestre et évoquant l'image de l'union de l'âme et du corps avant leur dissociation. En somme, la «conquête imaginaire des espaces aériens» n'est autre qu'un moyen "pour vaincre le tragique» de la condition humaine (p. 169). L'auteur conclut sur le soin qu'Euripide met à décrire l'Éther dans les évocations du vol de Persée, qui devient une véritable substance dense et physique, provoquant un plaisir particulier lors du vol, avec une grande poésie. Elle l'érige comme principe pour souligner la vertu poïétique du texte: les sensations produites par la poésie des images doivent venir toucher les spectateurs par d'autres biais que l'intellect.

Lucie THÉvenet

Autour du "banquet». Modèles de consommation et usages sociaux, sous la direction d'Arianna Esposito, avec la collaboration d'Élisabeth Rabeisen et Stefan Wirth, Dijon, Éditions universitaires de Dijon (Sociétés), 2015, 440 pages.

Le titre de ce recueil d'articles, Autour du «banquet», est une invitation au partage des connaissances sur une pratique sociale - la commensalité - à travers la diversité 